\title{
Introduction to Special Issue on Energy Finance
}

\section{Loretta Mastroeni ${ }^{1} \cdot$ Ralf Wunderlich ${ }^{2}$}

/ Accepted: 27 October 2021 / Published online: 13 November 2021

(C) The Author(s), under exclusive licence to Associazione per la Matematica Applicata alle Scienze Economiche e Sociali (AMASES) 2021

This special issue of Decisions in Economics and Finance was driven by the 2020Energy Finance Workshop held at the Roma TRE University in Rome, but it is important to note that acceptance at the workshop was neither a sufficient nor a necessary condition for acceptance to the Special Issue of the Journal. Indeed, twenty-one submissions were processed and, after blind peer reviews, ten papers were selected and presented in this volume.

It is interesting to note that in most of these ten papers, extensive use is made of machine learning, artificial intelligence and other updated computational techniques. In particular, five of the presented papers address issues such as

- gas storage optimization;

- investigation of the complex dynamics of electricity prices in power markets;

- valuation of derivatives written on agricultural commodities;

- investigation of the role of structural variables to explain the ESG Bloomberg ratings;

- studying of the impact of Clean Spark Spread expectations on storage hydroelectric generation.

Another two papers exploit optimal control techniques, one with a view to studying optimal adoption of an electric vehicle, while the second one uses those techniques to implement the solution of the optimal installation of renewable power production devices.

As for the remaining three papers, one investigates the effects of responsible investments on the volatility of European stock returns, while, in the second one, suitable multivariate Lévy processes are constructed to model the propagation of the system-

Loretta Mastroeni

lmastroeni@os.uniroma3.it; loretta.mastroeni@uniroma3.it

Ralf Wunderlich

ralf.wunderlich@b-tu.de

1 Department of Economics, Roma Tre University, Via Silvio D’Amico 77, 00145 Rome, Italy

2 Institute of Mathematics Brandenburg, University of Technology Cottbus-Senftenberg, Platz der Deutschen Einheit 1, 03046 Cottbus, Germany 
atic risk in dependent markets. In the third paper, the author proposes a new model for the pricing of wind power futures written on the wind power production index.

Hereafter, there will be a brief description of each paper.

\section{Machine learning, artificial intelligence and other computational tech- niques}

1. A deep learning model for gas storage optimization (N. Curin, M. Kettler, X. Kleisinger-Yu, V. Komaric, T. Krabichler, J. Teichmann, H. Wutte)

In this article, the authors utilize techniques inspired by reinforcement learning in order to optimize the operation plans of underground natural gas storage facilities. They provide a theoretical framework and assess the performance of the proposed method numerically in comparison with a state-of-the-art least-squares Monte Carlo approach. Due to the inherent intricacy originating from the highdimensional forward market as well as the numerous constraints and frictions, the optimization exercise can hardly be tackled by means of traditional techniques.

2. Gaussian clustering and jump-diffusion models of electricity prices: A deep learning analysis (C. Mari, E. Mari)

The authors propose a deep learning-based methodology to investigate the complex dynamics of electricity prices observed in power markets. The aims are: (i) to treat missing data in power price time series with irregular observation times; (ii) to detect a Gaussian component in the log-return empirical distributions if there is any; and (iii) to define suitable stochastic models of the power price dynamics. They apply this methodology to the US wholesale electricity price time series that are characterized by missing data, high volatility, jumps and spikes. To this end, a multilayer neural network is built and trained on a dataset containing information on market prices, traded volumes, numbers of trades and counterparties. The forecasts of the trained neural network are used to fill the gaps in the electricity price time series. Starting from the no-gap reconstructed electricity price time series, clustering techniques are then used to identify the largest Gaussian cluster in the log-return empirical distribution. They found that in each market under investigation log-returns show very large Gaussian clusters. This fact allows to decouple normal stable periods in which log-returns show a Gaussian behavior from turbulent periods in which jumps and spikes can occur. The decoupling between the stable motion and the turbulent motion allows to define suitable mean-reverting jump-diffusion models of power prices and to provide an estimation procedure that makes use of the full information contained in both the Gaussian component and the jumpy component of the log-return distribution. The obtained results show an interesting agreement with empirical data.

3. A machine learning-based price state prediction model for agricultural commodities using external factors (P. Octoviany, R. Knobloch, R. Korn)

In recent times of noticeable climate change, the consideration of external factors, such as weather and economic key figures, becomes even more crucial for a proper valuation of derivatives written on agricultural commodities. The occurrence of remarkable price changes because of severe changes in these factors motivates the 
introduction of different price states, each describing different dynamics of the price process. To include external factors, the authors propose a two-step hybrid model based on machine learning methods for clustering and classification. First, they assign price states to historical prices using K-means clustering. These price states are also assigned to the corresponding data of external factors. Second, predictions of future price states are then obtained from short-term predictions of the external factors by means of either K-nearest neighbors or random forest classification. They apply their model to real corn futures data and generate price scenarios via a Monte Carlo simulation. Therefore, the authors obtain a better approximation of the real futures prices by the simulated futures prices regarding the error measures MAE, RMSE and MAPE. From a practical point of view, these simulations can be used to support the assessment of price risks in risk management systems or as decision support regarding trading strategies under different price states.

4. Fundamental ratios as predictors of ESG scores: a machine learning approach (V. D’Amato, R. L. D’Ecclesia, S. Levantesi)

Sustainable and responsible finance incorporates Environmental, Social and Governance (ESG) principles into business decisions and investment strategies. In recent years, investors have rushed to sustainable and responsible investments in response to growing concerns about the risks of climate change. Asset managers look for some assessment of sustainability for guidance and benchmarking; for instance, 30 trillion of assets are invested using some ESG ratings. Several studies argue that good ESG ratings helped to prop up stock returns during the 2008 Global Financial Crisis. The ESG score represents a benchmark of disclosures on public and private firms, and it is based on different characteristics which are not directly related to the financial performance. The role of ESG ratings and their reliability has been widely discussed. Sustainable investment professionals are dissatisfied with climate disclosure by publicly traded companies. This negative sentiment is particularly strong in the USA, and within asset managers who do not believe that markets are consistently and correctly pricing climate risks into company and sector valuations. The authors believe that the ESG ratings, when available, still affect business and finance strategies. ESG ratings may represent a crucial element in the company's fund-raising process and on shares returns, and the main issue is how accurate are the available ESG ratings? The authors aim to assess how structural data as balance sheet items for traded companies affect ESG scores. Using the Bloomberg ESG scores, they investigate the role of structural variables adopting a machine learning approach, in particular the random forest algorithm. Precisely they use balance sheet data for the constituents of Euro Stoxx 600 index, referred to the last decade, and investigate how these explain the ESG Bloomberg ratings. They find that balance sheet items represent a powerful tool to explain the ESG score.

5. The impact of Clean Spark Spread expectations on storage hydropower generation (C. Condemi, L. Mastroeni, P. Vellucci) https://doi.org/10.1007/s10203-021-00355-6

Storage hydropower generation plays a crucial role in the electric power system and energy transition because it is the most widespread power generation with 
low greenhouse gas emissions, and moreover, it is relatively cheap to ramp up and down. As a result, it provides flexibility to the grid and helps mitigate the shortterm production uncertainty that affects most green energy technologies. However, using water in reservoirs represents an opportunity cost, which is related to the evolution of plant production capacity and production profitability. As the latter is related to a wide range of types of variables, to incorporate it in a large-scale prediction model, it is important to select the variables that impact most on storage hydropower generation. In this paper, the authors investigate the impact of the variables influencing the choices of price maker producers, and in particular, they study the impact of Clean Spark Spread expectations on storage hydroelectric generation. In this connection, using entropy and machine learning tools, they present a method for embedding these expectations in a model to predict storage hydropower generation, showing that, for some time horizon, expectations on CSS have a greater impact than expectations on power prices. It is shown that if the right mix of power price and CSS expectations is considered, the prediction error of the model is drastically reduced. This implies that it is important to incorporate CSS expectations into the storage hydropower model.

\section{Optimal control theory applications}

6. Optimal Switch from a Fossil-Fueled to an Electric Vehicle (P. Falbo, G. Ferrari, G. Rizzini, M.D. Schmeck)

In this paper, the authors propose and solve a real options model for the optimal adoption of an electric vehicle. A policymaker promotes the abeyance of fossilfueled vehicles through an incentive, and the representative fossil-fueled vehicle's owner decides the time at which buying an electric vehicle, while minimizing a certain expected cost. This involves a combination of various types of costs: the stochastic opportunity cost of driving one unit distance with a traditional fossilfueled vehicle instead of an electric one, the cost associated with traffic bans and the net purchase cost. After determining the optimal switching time and the minimal cost function for a general diffusive opportunity cost, the authors specialize to the case of a mean-reverting process. In such a setting, they provide a model calibration on real data from Italy and study the dependency of the optimal switching time with respect to the model's parameters. Moreover, they study the effect of traffic bans and incentive on the expected optimal switching time. They observe that incentive and traffic bans on fossil-fueled transport can be used as effective tools in the hand of the policymaker to encourage the adoption of electric vehicles, and hence to reduce air pollution.

7. Optimal installation of renewable electricity sources: the case of Italy (A. Awerkin, T. Vargiolu)

The authors test the real impact of current renewable installed power in the electricity price in Italy and assess how much the renewable installation strategy put in place in Italy deviated from the optimal one obtained from the model in the period 2012-2018. To do so, they consider the Ornstein-Uhlenbeck (OU) process, including an exogenous increasing process influencing the mean-reverting term, 
which is interpreted as the current renewable installed power. They estimate the parameters of this model by using real data of electricity prices and energy production from photovoltaic and wind power plants from the six main Italian price zones. They obtain that the model fits well the North, Central North and Sardinia zones: Among these zones, the North is impacted by photovoltaic production, Sardinia by wind and the Central North does not present significant price impact. Then, the authors implement the solution of the singular optimal control problem of installing renewable power production devices, in order to maximize the profit of selling the produced energy in the market net of installation costs. They extend the results already in the literature to the case in which no impact on power price is presented, and to the case in which $\mathrm{N}$ players can produce electricity by installing renewable power plants. To this extent, they analyze both the concepts of Pareto optima and of Nash equilibria. For this latter, the authors present a verification theorem in the 2-player case, and an explicit characterization of a Nash equilibrium in the no-impact case. We are thus able to describe the optimal strategy and compare it with the real installation strategy that was put in place in Italy.

\section{Energy and financial markets}

\section{Responsible Investments Reduce Market Risks (G. Morelli, R. L. D'Ecclesia)}

Responsible investments are considered as one of the driving factors of revenues growth enhancing risk-adjusted returns. This paper investigates the effects of responsible investments on the volatility of European stock returns. First, the authors exploit an expectation-maximization (EM) algorithm to cluster the companies into two groups according to the environmental score (E), used as a proxy for responsible investments. Second, they build one global minimum variance portfolio (GMV) within each group and estimate its volatility using ARCH-type models. Finally, they forecast well-known risk measures such as the value at risk (VaR) and the expected tail loss (ETL) to assess market risks for investing green. Responsible portfolios composed of stocks with high E score outperform their low E counterparts and are shown to be safer choices to mitigate risks, especially during periods of market distress. The results are remarkable for many sectors.

9. A New Approach to Wind Power Futures Pricing (M. Hess)

https://doi.org/10.1007/s10203-021-00345-8

The author proposes a new model for the pricing of wind power futures written on the wind power production index. His approach is based on an arithmetic multifactor pure-jump Ornstein-Uhlenbeck setup with time-dependent coefficients and expresses the wind power production index and the corresponding futures price in terms of Fourier integrals to derive the related time dynamics. The paper closes with an investigation of the so-called risk premium associated with the treated wind power model.

10. Correlating Lévy processes with Self-Decomposability: Applications to Energy Markets (M. Gardini, P. Sabino, E. Sasso) https://doi.org/10.1007/s10203-021-00352-9 
Based on the concept of self-decomposability, the authors extend some recent multi-dimensional Lévy models built using multivariate subordination. Their aim is to construct multivariate Lévy processes that can model the propagation of the systematic risk in dependent markets with some stochastic delay instead of affecting all the markets at the same time. To this end, they extend some known approaches keeping their mathematical tractability, study the properties of the new processes, derive closed-form expressions for their characteristic functions and detail how Monte Carlo schemes can be implemented. The authors illustrate the applicability of their approach in the context of gas, power and emission markets focusing on the calibration and on the pricing of spread options written on different underlying commodities.

Acknowledgements We would like to thank the Editor-in-Chief Paolo Ghirardato for the opportunity to publish this Special Issue. Besides, we would like to thank all the authors who contributed to it and the anonymous referees for their precious work.

Publisher's Note Springer Nature remains neutral with regard to jurisdictional claims in published maps and institutional affiliations. 